\title{
Hepatic Encephalopathy: Pharmacological Therapies Targeting Ammonia
}

\author{
Robert S. Rahimi, MD, MS ${ }^{1}$ Don C. Rockey, MD² \\ ${ }^{1}$ Division of Hepatology, Department of Internal Medicine, Annette C. \\ and Harold C. Simmons Transplant Institute, Baylor University \\ Medical Center, Dallas, Texas \\ 2 Department of Internal Medicine, Medical University of South \\ Carolina, Charleston, South Carolina
}

Address for correspondence Robert S. Rahimi, MD, MS, Baylor University Medical Center, 3410 Worth Street, Suite 860, Dallas, TX 75246 (e-mail: robert.rahimi@baylorhealth.edu).

Semin Liver Dis 2016;36:48-55.

\author{
Abstract \\ Keywords \\ - AST-120 \\ - cirrhosis \\ - glycerol \\ phenylbutyrate \\ - lactulose \\ - molecular adsorbent \\ recirculating system \\ - ornithine \\ phenylacetate \\ - benzoate \\ - polyethylene glycol
}

Hepatic encephalopathy (HE) is a major complication in patients with decompensated cirrhosis, leading to higher readmission rates causing a profound burden of disease and considerable health care costs. Because ammonia is thought to play a crucial role in the pathogenesis of $\mathrm{HE}$, therapies directed at reducing ammonia levels are now being aggressively developed. Ammonia scavengers such as AST-120 (spherical carbon adsorbent), glycerol phenylbutyrate, sodium phenylacetate or sodium benzoate, and ornithine phenylacetate have been used to improve HE symptoms. A new approach, bowel cleansing with polyethylene glycol 3350 , appears to be a promising therapy, with a recent study demonstrating a more rapid improvement in overt HE (at 24 hours after treatment) than lactulose. Extracorporeal devices, although now used primarily in research settings, have also been utilized in patients with refractory $\mathrm{HE}$, but are not approved for clinical management.
One of the most common and dreaded complications in patients with cirrhosis is hepatic encephalopathy (HE). Those with HE can experience a spectrum of altered mentation that ranges from covert $\mathrm{HE}$ (previously termed minimal $\mathrm{HE}$ ) to overt HE (previously termed acute $H E$ ), and accounts for decreased quality of life, higher readmission rates, and poorer outcomes than in cirrhotic patients who have not experienced any HE episodes. ${ }^{1}$

Although HE leads to altered cerebral function, primarily hypothesized to be due to elevated ammonia production by gut bacteria, the pathophysiology of HE should be considered to be multifactorial in etiology (-Fig. 1) in which the underlying mechanisms are still not well understood. ${ }^{2}$ For one, the production of ammonia can arise from gut bacteria (i.e., Clostridium, Enterobacter, and Proteus species) that convert urea from the blood into ammonia and carbon dioxide via urease. $^{3-5}$ Additionally, enterocytes in the small intestine also appear to generate ammonia, metabolizing glutamine into glutamate and ammonia via glutaminase, ${ }^{6,7}$ which has been demonstrated to be upregulated in cirrhotic patients with covert and overt HE (- Figs. 1 and 2) ${ }^{8,9}$ Furthermore, because the ability of the liver to metabolize ammonia effectively is diminished in cirrhosis, and portal hypertension leads to shunting of blood around the sinusoids, hyperammonemia ensues, resulting in astrocyte dysfunction, triggering neurologic abnormalities. ${ }^{10}$ Irrespective of the precise mechanism of hyperammonemia, because ammonia is thought to play a major pathophysiologic role in $\mathrm{HE}$, in this review we will focus on ammonia-lowering approaches, in particular novel ones.

\section{Ammonia Scavengers}

Sodium benzoate and sodium phenylacetate have been approved for use as ammonia scavengers in patients with urea cycle disorder (UCD) and hyperammonemia (typically seen in children). These compounds are water soluble, and help eliminate ammonia through the urine (-Table 1; - Fig. 1). Oral sodium benzoate (Ucyclyd Pharma, Inc.) is sometimes used off-label for ammonia scavenging and is available in
Issue Theme New Treatments in Liver Disease; Guest Editors, Gregory J. Gores, $\mathrm{MD}$, and Ariel E. Feldstein, MD
Copyright @ 2016 by Thieme Medical Publishers, Inc., 333 Seventh Avenue, New York, NY 10001, USA. Tel: +1(212) 584-4662. 


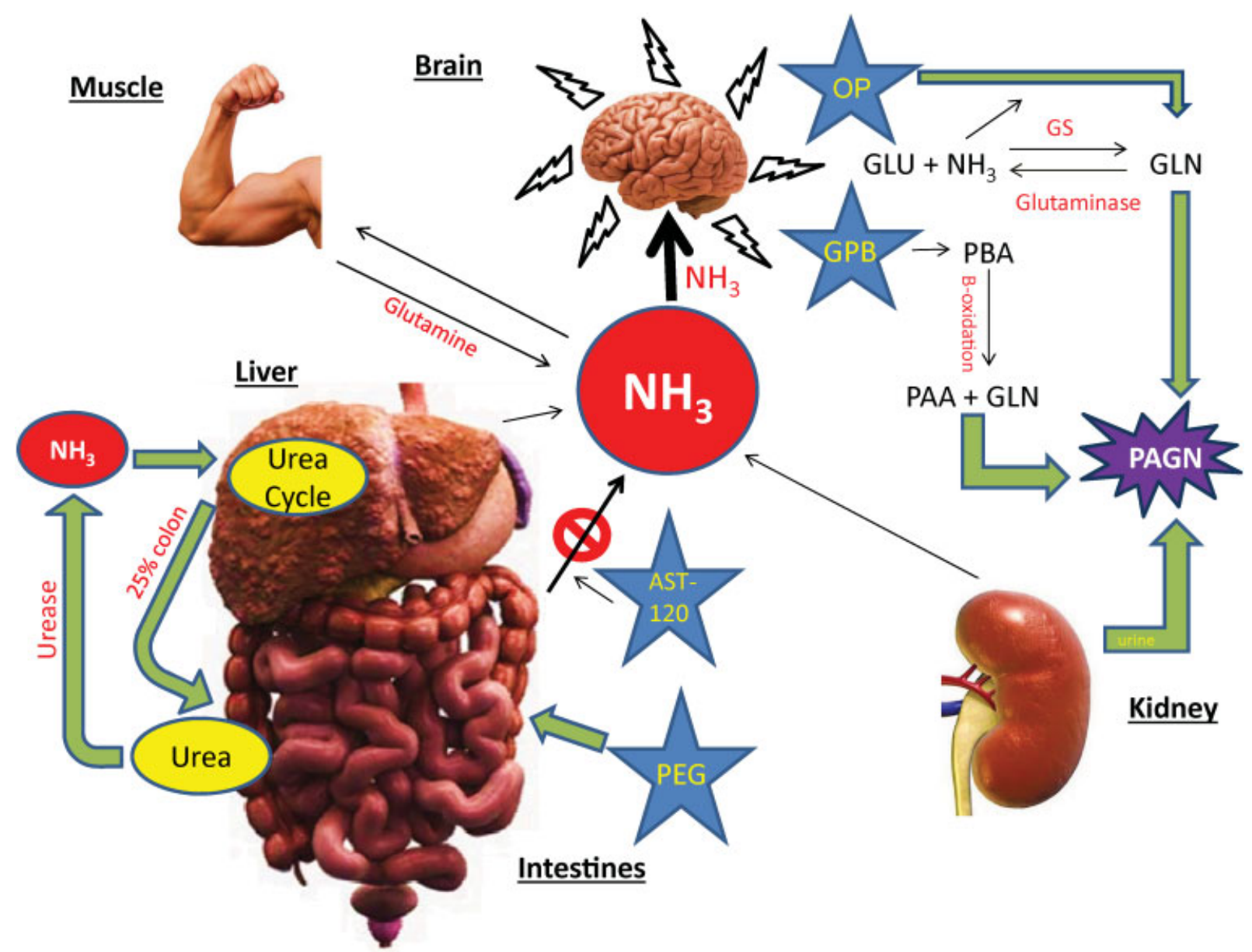

Fig. 1 Multiorgan ammonia pathways with specific ammonia lowering medications used in cirrhosis. Circulating concentrations of ammonia $\left(\mathrm{NH}_{3}\right)$ are shown with multiorgan involvement in the production of $\mathrm{NH}_{3}$, ultimately resulting in $\mathrm{NH}_{3}$ crossing the blood-brain barrier, contributing to astrocyte swelling and hepatic encephalopathy; as decreased urea cycle capability and reduced liver glutamine synthetase activity is present in cirrhosis. The alternative pathway is also shown at the top, where $\mathrm{NH}_{3}$ binds with glutamate (GLU) forming glutamine (GLN) after enzymatic processing using glutamine synthetase (GS). Both ornithine phenylacetate (OP) and glycerol phenylbutyrate (GPB) are ammonia-lowering medications; they combine GLN and phenylacetate (PAA) to form phenylacetylglutamine (PAGN), which is excreted in the urine. AST-120 is a carbon microsphere adsorbent that binds $\mathrm{NH}_{3}$ in the gut, thus lowering circulating ammonia levels. Polyethylene glycol (PEG) is a cathartic, which causes rapid clearance of gut bacterial synthesizing ammonia to be excreted into the feces. About one-quarter of urea-derived byproducts from the urea cycle is shunted to the colon (not shown; remaining three-fourths of urea excreted in the kidneys), where urease producing bacterial organisms produce ammonia that enters the portal circulation. Skeletal muscle also contributes in the regulation of $\mathrm{NH}_{3}$ as depicted. $\mathrm{Not}$ shown in the figure is the presence of GS and glutaminase in each organ, contributing to $\mathrm{NH}_{3}$ homeostasis. PBA = phenylbutyric acid.

powder form from certain specialty pharmacies. Although these pharmacological therapies are available in the United States, they are not approved for HE. Several considerations related to large therapeutic doses of these agents are noteworthy. First, they lead to a high sodium load (1-2 g/d for therapeutic dosing), which might lead to hypernatremia and/ or fluid retention in cirrhotic patients. Furthermore, these products are less palatable (i.e., salty taste) compared with newer agents such as glycerol phenylbutyrate (GPB; HPN100; Ravicti; Horizon Pharma, Plc.), which is nearly tasteless, is safe, and well tolerated. ${ }^{11,12}$

\section{Glycerol Phenylbutyrate}

Glycerol phenylbutyrate improves the organoleptic properties of its predecessor, a prodrug of phenylacetate, oral sodium phenylbutyrate (Buphenyl, Horizon Pharma, Plc.) and is a nearly tasteless, odorless, sodium-free liquid compound, whose mechanism of action is removal of nitrogen excretion in the form of urinary phenylacetylglutamine (PAGN), allowing an alternative pathway for ammonia waste ( - Figs. 1 and $\mathbf{2}$ ). ${ }^{13}$ It is approved in the treatment of UCD, an inherited disorder causing hyperammonemia. ${ }^{14-17}$

Because GPB has demonstrated ammonia-lowering capabilities, an open-label pilot study assessed the effect on venous ammonia and the tolerability in cirrhotic patients with HE (15 patients given $6 \mathrm{~mL}$ vs. $9 \mathrm{~mL}$ of GPB orally twice daily with food for 4 weeks). ${ }^{11}$ Compared with baseline ammonia concentrations, fasting ammonia levels were lower on GPB on the eighth day of $6 \mathrm{~mL}$ twice daily dosing to 45.4 (27.9) $\mu \mathrm{mol} / \mathrm{L}$ (upper limit of normal $\sim 48 \mu \mathrm{mol} / \mathrm{L} ; p<0.05$ ). Using $9 \mathrm{~mL}$ twice daily yielded similar ammonia lowering levels, but was associated with higher phenylacetate (PAA) plasma concentrations and more adverse events. 


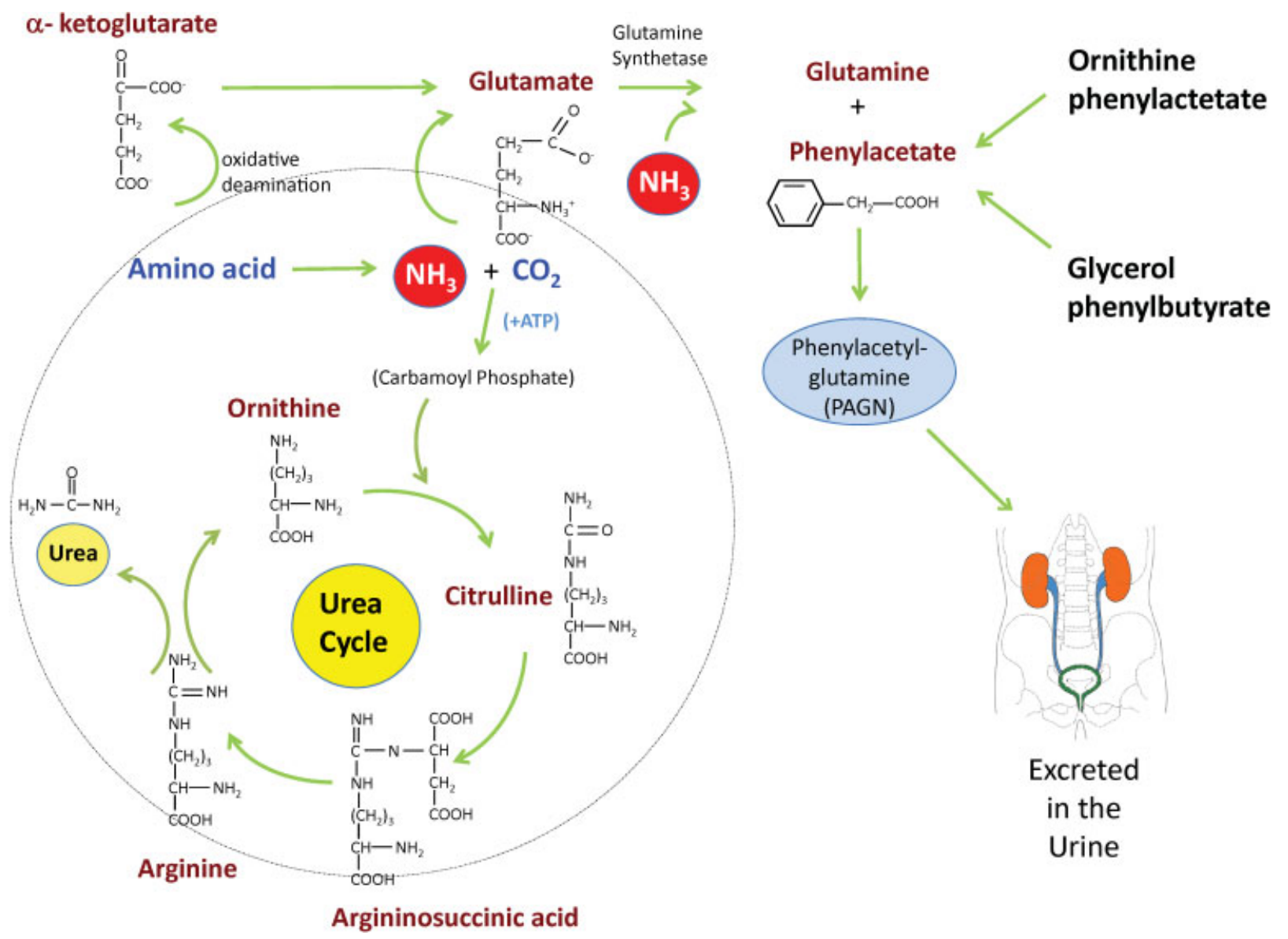

Fig. 2 Ammonia metabolism by the urea (ornithine) cycle and alternate ammonium excretion pathways. Ammonia is the product of oxidative deamination reactions, and is an end product of amino acid/protein metabolism. Thus, ammonia is a waste product, which is normally converted to urea and excreted in the urea cycle as depicted within the dashed circle. The addition of ammonia $\left(\mathrm{NH}_{3}\right)$ to the urea cycle, results in urea and ornithine formation. In patients with liver disease and hepatocellular dysfunction, enzymatic processes in dysfunctional hepatocytes are presumably unable to handle $\mathrm{NH}_{3}$, resulting in accumulation of $\mathrm{NH}_{3}$. In the upper portion of the figure, an alternative pathway exists in which glutamine synthetase uses ammonia and glutamate as substrates to form glutamine. Glutamine then combines with phenylacetate to form phenylacetylglutamine (PAGN). Ornithine phenylacetate and glycerol phenylbutyrate provide phenylacetate as substrate (glycerol phenylbutyrate is metabolized to phenylbutyric acid in the intestine, and undergoes $\beta$-oxidation in the liver and other organs to phenylacetate). Phenylacetate and glutamine (by the action of glutamine $\mathrm{N}$-acyltransferase) are combined to form PAGN, which is harmlessly excreted into the urine; this alternative pathway allows ammonia and excess nitrogen waste to be excreted from the body.

This study therefore led to a multicenter, randomized, double-blind, placebo-controlled phase II trial to test the hypothesis that using GPB for ammonia lowering $(6 \mathrm{~mL}$ twice daily for 16 weeks in patients with $\mathrm{HE}$ ), could decrease the incidence of HE events in cirrhotic patients who demonstrate a history of at least two HE episodes $\geq$ grade 2 within the past 6 months, including one $\mathrm{HE}$ event within 3 months of randomization (Clinicaltrials.gov, NCT00999167). ${ }^{12}$ Exclusion criteria included the following: use of other ammonia-lowering agents (e.g., Lornithine-L-aspartate or sodium benzoate), active complications of cirrhosis (e.g., sepsis, hepatocellular carcinoma, hepatic hydrothorax), gastrointestinal (GI) bleeding requiring blood transfusion within 3 months, transjugular intrahepatic portosystemic shunt placement or revision within 90 days, recreational drug use or alcohol consumption for patients with a history of alcohol or drug abuse within 6 months, regular use of benzodiazepines, narcotics, or barbiturates, prolonged QT interval, Model for End Stage Liver Disease (MELD) score $>25$, serum creatinine $>2 \mathrm{mg} / \mathrm{dL}$, serum sodium $<125 \mathrm{mEq} / \mathrm{L}$, platelet count $<35,000 / \mu \mathrm{L}$, hemoglobin $<8 \mathrm{~g} / \mathrm{dL}$, hematocrit $<25 \%$, expected liver transplantation within 6 months, and hypersensitivity to GPB or its metabolites. It is notable that patients taking rifaximin were eligible if they had been on a stable dose for at least 1 month, and had experienced at least one of their two qualifying HE events while taking rifaximin.

There were 178 patients enrolled; 55 of 90 (61\%) patients in the GPB arm and 67 of 88 (76\%) patients in the placebo arm completed therapy. Baseline characteristics, including lactulose use were similar between the two groups; however, less Child-Pugh class $C$ patients were randomized to placebo than GPB ( 8 vs. 21 patients, respectively), and more men were randomized to placebo than to GPB (59 vs. 45 patients) with only U.S. patients using rifaximin at baseline. In the intention to treat (ITT) groups, 19 of 35 (54\%) patients in the GPB arm versus 16 of 21 (76\%) patients in the placebo arm met predetermined stopping rules, and therefore discontinued therapy in each group, respectively. In the ITT population, a higher proportion of patients in the placebo group compared 


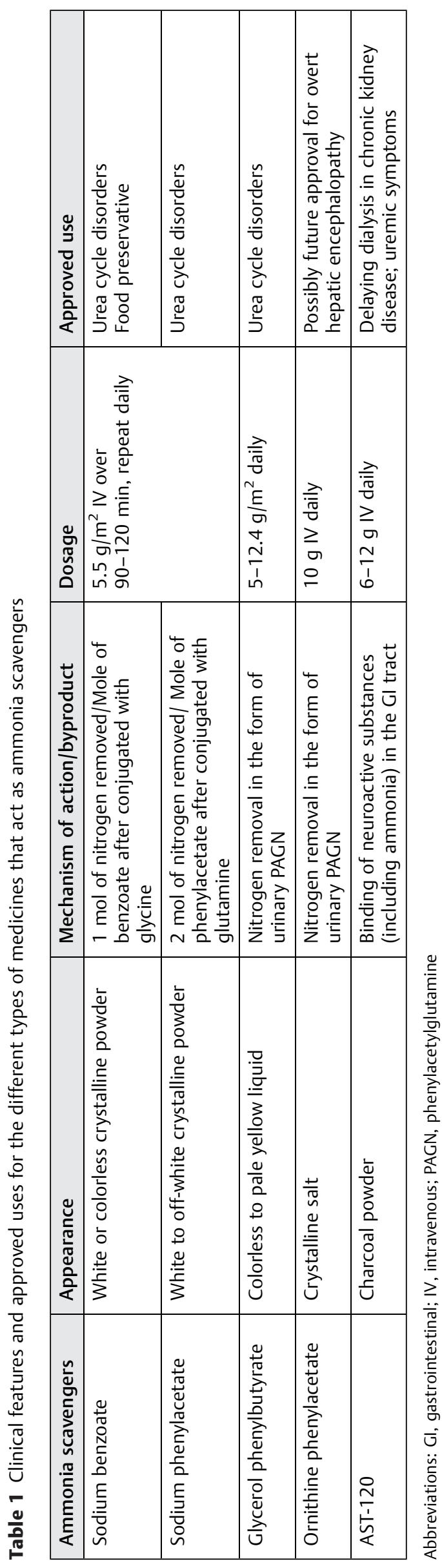

with the GPB group experienced an HE event (36\% vs. $21 \%$; $p=0.02$ ), meeting the primary endpoint. Furthermore, time to first event (hazard ratio $[\mathrm{HR}]=0.56 ; p<0.05$ ), total events ( 35 vs. $57 ; p=0.04$ ), and fewer HE hospitalizations (13 vs. $25 ; p=0.06$ ) occurred in the GPB versus placebo, respectively. Among patients not on rifaximin at enrollment, GPB demonstrated more efficacy, reducing the proportion of patients with an HE event (10\% vs. $32 \% ; p<0.01$ ), time to first event $(\mathrm{HR}=0.29 ; p<0.01)$, and total events ( 7 vs. 31 ; $p<0.01$ ). Plasma ammonia levels were significantly lower in patients on GPB, which correlated with HE events when measured either at baseline or during the study. Nearly $75 \%$ in both groups reported similar adverse events.

The data suggest that GPB decreased the likelihood of hospitalization for cirrhotic patients with recurrent $\mathrm{HE}$ when compared with placebo, by lowering ammonia levels. Overall, GPB was considered to be safe among cirrhotic patients with recurrent HE; however, larger randomized trials are needed to further establish the role of GPB in patients with HE.

\section{Ornithine Phenylacetate}

Ornithine phenylacetate (OP; OCR-002) has been shown to decrease ammonia by combining ornithine and phenylacetate in a crystalline salt to enhance the disposal of nitrogenous molecules in urine. First, L-ornithine acts as a substrate for glutamine synthesis from ammonia in skeletal muscle; second, phenylacetate enhances the excretion of the ornithine-related glutamine as phenylacetylglutamine (PAGN) in the kidney (-Figs. 1 and 2). ${ }^{18}$ During a 5-day continuous infusion in cirrhotic patients with upper gastrointestinal bleeding (UGIB), the safety (plasma and urine metabolites) and tolerability of OP was studied. ${ }^{19}$ The investigators enrolled 10 of 26 (38\%) cirrhotic patients with UGIB and matched them for plasma creatinine and Child-Pugh stage to cirrhotic patients that received OP. Exclusion criteria included the following: terminal illness, need for mechanical ventilation, infection by human immunodeficiency virus, signs of HE including neurologic comorbidities that might impair mental status, creatinine $>1.5 \mathrm{mg} / \mathrm{dL}$, or need for hemodialysis, suspected allergic reaction to the drug components, or electrocardiogram with QT interval corrected Fridericia (QTcF) $>500 \mathrm{~ms}$ (Bazzet formula). Patients taking the following drugs were also excluded: systemic corticosteroids, valproic acid, haloperidol, penicillin, and probenecid. The initial OP dose was $0.138 \mathrm{~g} / \mathrm{h}$, then increased to $0.275 \mathrm{~g} / \mathrm{h}$ after 12 hours and to $0.416 \mathrm{~g} / \mathrm{h}$ after 24 hours, with incremental dose adjustments if patients did not develop predefined stopping criteria. All 10 patients completed the study achieving a target dose of $10 \mathrm{~g} / \mathrm{d}$ of intravenous OP over 5 days. The mean $(M)$ age was 60 years, 9 of 10 (90\%) patients were male, with Child-Pugh class A: 7/10 (70\%); Child-Pugh class B: $3 / 10$ (30\%), 6/10 (60\%) Laennec's, 2/10 (20\%) hepatitis C, $1 / 10$ (10\%) hepatitis B, and $1 / 10$ (10\%) cryptogenic cirrhosis. Plasma ammonia was significantly higher in the control group at 24 hours and showed a progressive decline between baseline $(80 \pm 43 \mu \mathrm{mol} / \mathrm{L}), 36$ hours $(42 \pm 15 \mu \mathrm{mol} / \mathrm{L}), 72$ 
hours ( $44 \pm 15 \mu \mathrm{mol} / \mathrm{L}), 96$ hours $(40 \pm 24 \mu \mathrm{mol} / \mathrm{L})$, and 120 hours ( $33 \pm 14 \mu \mathrm{mol} / \mathrm{L}$ ). Plasma glutamine also decreased ( $-37 \%$ at day 5$)$, with its excretion in urine as PAGN progressively rising ( $52 \pm 35 \mathrm{mmol}$ at day 5 ). No serious adverse events were reported. Currently, OP is under clinical development in decompensated cirrhosis for acute $\mathrm{HE}$, with phase IIb study results expected in 2017. This compound thus may prove to be useful to treat overt HE episodes. ${ }^{20}$

\section{AST-120}

AST-120 (spherical carbon adsorbent; Ocera Therapeutics) is an orally engineered carbon microsphere adsorbent $(0.2-$ $0.4 \mathrm{~mm}$ in diameter) with a highly nonspecific surface area $\left(>1,600 \mathrm{~m}^{2} / \mathrm{g}\right.$ ), approved in Japan in 1991, which has been used to improve uremia and delay the initiation of dialysis in patients with chronic renal insufficiency. ${ }^{21-24}$ AST-120 differs structurally from activated charcoal in that it exhibits superior adsorption from the lumen of the lower GI tract for certain organic compounds (i.e., low molecular weight $<10 \mathrm{kDa}$ ) due to its unique nanopore volume and size, allowing for a selective binding surface (- Fig. 1). ${ }^{25}$ Minimal interference with digestive enzymes or drug absorption is seen because AST-120 is not degraded or adsorbed from the GI tract, rendering it safe for prolonged use.

In portocaval shunted dogs, AST-120 was shown to lower plasma ammonia levels, ${ }^{26}$ and preliminary studies in humans with low-grade HE demonstrated neurocognitive benefits. ${ }^{27}$ Previously, AST-120 was shown to bind a variety of neuroactive substances linked to the pathogenesis of HE, thereby lowering ammonia levels and reducing brain edema. AST-120 was shown to decrease oxidative stress, locomotor activity, brain edema, and arterial ammonia in rats induced with secondary biliary cirrhosis after bile-duct ligation (BDL). ${ }^{28}$ In in vitro assessments of AST-120, ammonia was adsorbed in similar fashion as the human GI tract physiologic $\mathrm{pH}^{29,30}$; with $60 \%$ adsorption of ammonia within the first hour and 77 to $94 \%$ adsorption occurring after 6 hours of incubation at different concentrations. AST-120 was administered to both $\mathrm{BDL}$ and sham control rats in vivo for different time frames at different doses. In the group receiving the compound for 6 week prior to injury, arterial ammonia was increased in nontreated BDL rats compared with sham controls $(177.3 \pm 30.8 \mu \mathrm{M}$ vs. $66.5 \pm 18.2 \mu \mathrm{M}, p<0.01)$, although all doses of AST-120 decreased ammonia in a dose-dependent manner in BDL rats compared with levels found in sham controls (at using concentrations of $0.1,1$, and $4 \mathrm{~g} / \mathrm{kg} / \mathrm{d}$, respectively). Ammonia levels correlated with the dose of AST-120 (Spearman $r=-0.6603 ; p=0.0006$ ) and were significantly lower in treated BDL versus nontreated BDL rats $(p<0.01)$. Brain water content in all portions of the brain were significantly increased in nontreated BDL rats versus nontreated sham controls, and AST-120 led to reduced brain water content in all areas studied. The effect of AST-120 in short-term treatment regimens (3 days or 2 weeks after surgery), also significantly reduced brain water content and arterial ammonia levels in all brain regions, in comparison with nontreated BDL animals, relative to sham-operated controls $(p<0.05)$. Locomotor activity in BDL rats was decreased compared with sham controls, but normalized after AST-120 therapy. Finally, AST-120 abrogated the increase in blood ammonia after intravenously infused ammonium acetate, thereby precluding progression to precoma in BDLtreated rats in comparison to nontreated rats $(p<0.05)$.

A double-blind, randomized, multicenter placebo controlled trial examined the overall safety, tolerability, and effectiveness of AST-120 (given for 8 weeks) in 148 patients with compensated cirrhosis (with MELD scores $<25$ ) and covert hepatic encephalopathy (CHE). ${ }^{31}$ The majority of patients (53\%) had chronic hepatitis $\mathrm{C}$, with an average MELD of 10. Patients were randomized to receive $6 \mathrm{~g}$ AST120 daily $(n=50), 12$ g AST-120 daily $(n=50)$, or placebo $(n=48)$. Baseline psychometric scores, clinical global assessment of $\mathrm{HE}$ or overt $\mathrm{HE} /$ hospitalizations between all groups were similar. Independent of neurocognitive improvement, venous ammonia decreased significantly from baseline after 8 weeks of treatment in both AST-120 groups compared with placebo. The authors suggested that although AST-120 therapy did not meet the primary endpoint of improvement in the Repeatable Battery for the Assessment of Neuropsychological Status (RBANS) due to a confounding study design allowing improvement in neurocognitive measures before randomization, AST-120 was well tolerated. The final phase II study results have not been reported in full form as of the current time.

\section{Molecular Adsorbent Recirculating System and Bioartificial Devices}

Extracorporeal nonbiologic liver support devices that remove ammonia from the body are appealing therapeutic alternatives for patients with $\mathrm{HE}$, especially patients with liver failure awaiting liver transplantation. Although several of these "liver or albumin dialysis" systems are currently available, the molecular adsorbent recirculating system (MARS; Baxter Intl.) represents the most advanced device, which was granted Food and Drug Administration approval in January 2013 for the treatment of HE. It is used in over 45 countries worldwide, although mostly for research purposes. The MARS device has been assessed mostly in patients with refractory $\mathrm{HE}$, often in patients with acute liver failure. In patients with cirrhosis and refractory HE, the MARS system was studied in a large multicenter randomized controlled trial for patients with severe HE, not responding to standard treatment. ${ }^{32}$ The MARS system, used for 6 hours daily for 5 days or until the patient had $\geq 2$ grade improvement in HE, was compared with standard medical therapy (SMT). The primary endpoint was to evaluate the difference in improvement of HE between the two groups. Of the 70 patients with a median age of 53 and a MELD score of 32, 44\% were female, demonstrating severe HE (grade 3, 56\%; grade 4, 44\%). Patients were randomized to SMT alone $(n=31)$ or MARS + SMT $(n=39)$, with less improvement of HE grade in the SMT group $(M=19 \%)$ compared with MARS + SMT $(M=34 \%)$, $p=0.044$, and was reached at a less frequent and lower rate compared with the MARS + SMT group $(p=0.045)$. Overall MARS therapy was tolerated well with no unexpected 
adverse events; , however, no benefit regarding mortality was demonstrated between the two groups.

Other devices, including bioartificial machines with hepatocytes, have been studied for the treatment of $\mathrm{HE}^{33}$ In one study, 18 patients with grade 2 to 4 acute HE that did not respond to at least 24 hours of medical therapy received a maximum of three 6-hour charcoal-based hemodiabsorption treatments. The mean time to improvement in $\mathrm{HE}$ grade $\leq 2$ or at least a $50 \% \mathrm{HE}$ index reduction was $2.6 \pm 1.9$ days and $89 \%(16 / 18)$ of patients had a response. Survival was $94 \%$ and $72 \%$ at 5 and 30 days. However, this and other similar therapies are not currently approved for use in the United States.

More recently, a systematic review of 10 trials ( 7 of which were MARS) was conducted, and although dialysis achieved a decrease in total serum bilirubin relative to SMT of $8 \mathrm{mg} / \mathrm{dL}$ (95\% confidence interval $[\mathrm{CI}]=-10.6$ to -5.4$)$, bile acids or serum ammonia levels were not significantly different. Although, HE grade did improve with MARS (risk ratio [RR] $=1.55 ; 95 \% \mathrm{CI}=1.16-2.08)$ compared with SMT overall survival was not significantly different $(\mathrm{RR}=0.95 ; 95 \% \mathrm{CI}$ $=0.84-1.07$ ) among the different treatments. There were no major safety concerns. The meta-analysis suggests that further studies are needed. ${ }^{34}$

\section{Polyethylene Glycol}

Because gut bacteria synthesize ammonia, it follows that their rapid clearance might result in a benefit in patients with HE. In fact, this has been our clinical experience with the use of polyethylene glycol (PEG). This led to a randomized clinical trial to study the effect of PEG in acute HE (Clinicaltrials.gov, NCT01283152) (- Fig. 1). ${ }^{35}$ In this study, in which 186 cirrhotic patients admitted for overt HE were screened, 50 patients met inclusion criteria: 25 patients received either a 4-L dose of PEG versus 25 patients receiving SMT with lactulose. The two groups were similar with respect to demographics, and laboratory and clinical features, with the exception of blood urea nitrogen (BUN), which was higher in the PEG group $(p=0.03)$. Seventy-eight percent of patients received one dose of lactulose per emergency department SMT protocol prior to randomization (lactulose arm, $n=19$; PEG arm, $n=20$ ), while the remaining $22 \%$ were recruited prior to lactulose administration. There were no significant differences among the groups with regard to the initial dosing with lactulose $(p=0.45)$. The primary outcome was improvement in at least $1 \mathrm{HE}$ grade using the hepatic encephalopathy scoring algorithm (HESA) within 24 hours for both groups. Initial HESA scores were identical $(M=2.3$, $p=0.70$ ) and there were no differences in the distribution of the scores $(p=0.62)$. Nine of $25(36 \%)$ subjects in the lactulose group had an incremental improvement of 1 HESA grade, 3 (12\%) improved by 2 grades, and $1(4 \%)$ improved by 3 HESA grades at 24 hours; 12 of 25 (48\%) subjects had no improvement. Only two patients had a HESA score of zero (8\%) at 24 hours. In contrast, 10 of $23(43 \%)$ subjects improved by 1 HESA grade at 24 hours, 9 (39\%) by 2 grades, and 2 by 3 grades (9\%); 2 of 23 (9\%) subjects receiving PEG had no improvement. Ten (43\%) subjects had a HESA score of zero at 24 hours. Overall, subjects receiving PEG had a significantly lower mean HESA score at 24 hours than patients receiving lactulose $(0.9 \pm 1.0$ vs. $1.6 \pm 0.9, p=0.002)$. The median time to $\mathrm{HE}$ resolution was 1 day in subjects receiving PEG compared with 2 days in receiving SMT lactulose $(p=0.01)$. Hospital length of stay was reduced in patients receiving PEG ( $4 \pm 3$ days) compared with controls ( $8 \pm 12$ days; $p=0.07$ ). There were no definitive adverse related events reported with either lactulose or PEG.

Regarding ammonia, excretion in the stool has been previously shown to be greater with PEG than with lactulose. ${ }^{36}$ Thus, an interesting observation in this study was that the 24-hour change in blood ammonia was greater in the lactulose group than in the PEG group; further, ammonia levels did not correlate with better improvement in HESA grades ( - Table 2). Possible explanations for this result could be due to the mechanism of action of the two study medications, ${ }^{36}$ or to the timing of the posttreatment ammonia level. Because PEG is a highly effective cathartic, the potential clinical improvement in HE might precede and be more clinically relevant than the actual decrease in ammonia levels, which would parallel the authors' hypothesis in this study. Alternatively, circulating ammonia levels may return to their elevated baseline faster (and before clinical deterioration is manifested). PEG may also in theory result in dehydration and decreased renal perfusion, which might lead to decreased renal ammonia excretion. Although ammonia levels were not a preplanned endpoint of the study, primary providers frequently obtained follow-up ammonia levels and these were reported on (this meant that the time between treatment initiation and blood collection varied). When the anion gap (AG) was analyzed separately, there were no statistically or clinically significant differences seen between lactulose and PEG (lactulose: AG before treatment $=12 \pm 3 \mathrm{mmol} / \mathrm{L}[\mathrm{M} \pm$ standard deviation (SD)], after treatment $=13 \pm 3, n=21$, $p=0.65, t$ test; PEG: AG before and after treatment $=14 \pm 3$, $n=22, p=0.86)$. When analyzed by treatment group and response; there were no meaningful differences in ammonia after treatment (lactulose: nonresponders initial ammonia $=147 \pm 74 \mu \mathrm{mol} / \mathrm{L}[M \pm \mathrm{SD}]$, follow-up ammonia $=75$ $\pm 31, n=8$; responders initial ammonia $=207 \pm 54$, follow-up ammonia $=90 \pm 26, n=7$; PEG: nonresponders initial ammonia $=74,139$, follow-up ammonia $=175,125$, $n=2$; responders initial ammonia $=172 \pm 93$, follow-up ammonia $=104 \pm 57, n=16$ ). The only statistically significant difference was that baseline ammonia levels were higher in lactulose responders than nonresponders $(p=0.044$, Spearman). ${ }^{37}$ Future studies evaluating ammonia and its correlation with HE resolution will likely help clarify this issue.

Given the theoretical possibility that PEG treatment may lead to alterations in electrolyte levels or renal function, this issue was also carefully assessed in this study. Electrolytes, creatinine, and BUN were measured at baseline and at 6 to 24 hours after admission. Potassium levels decreased from $4.3 \mathrm{mmol} / \mathrm{L}$ to 3.8 $\mathrm{mmol} / \mathrm{L}$ after PEG $(p=0.006)$. Six subjects in each the PEG and lactulose groups had moderate hypokalemia (potassium $<3.5$ $\mathrm{mmol} / \mathrm{L}$ ) during the first 6 to 24 hours after treatment. There 
54 Hepatic Encephalopathy: Pharmacological Therapies Targeting Ammonia Rahimi, Rockey

Table 2 Hepatic encephalopathy: lactulose versus polyethylene glycol 3350-electrolyte solution (HELP) study outcomes

\begin{tabular}{|l|l|l|l|l|}
\hline Variable & Total & Lactulose & PEG & $p$ Value \\
\hline 24-h change in HESA $(M \pm S D)$ & $1.1 \pm 0.8$ & $0.7 \pm 0.8$ & $1.5 \pm 0.8^{\mathrm{b}}$ & 0.002 \\
\hline 24-h change in ammonia & & & & \\
\hline Elapsed time $=6-24 \mathrm{~h}$ & $(n=33)$ & $(n=15)$ & $(n=18)$ & $146 \pm 75$ \\
\hline Before study Rx $(\mu \mathrm{mol} / \mathrm{L})$ & $159 \pm 73$ & $175 \pm 70$ & $120 \pm 60$ & 0.19 \\
\hline After study Rx $(\mu \mathrm{mol} / \mathrm{L})$ & $103 \pm 51$ & $82 \pm 29$ & $26 \pm 90$ & 0.049 \\
\hline Difference in level $(\mu \mathrm{mol} / \mathrm{L})$ & $56 \pm 88$ & $93 \pm 71$ & $4 \pm 3$ & 0.025 \\
\hline Length of stay & $6 \pm 9$ & $8 \pm 12$ & 4 & 0.07 \\
\hline
\end{tabular}

Source: From Rahimi RS, Singal AG, Cuthbert JA, Rockey DC. Lactulose vs polyethylene glycol 3350-electrolyte solution for treatment of overt hepatic encephalopathy: the HELP randomized clinical trial. JAMA Intern Med 2014;174(11):1727-1733.

Abbreviations: HESA, hepatic encephalopathy scoring algorithm; PEG, polyethylene glycol; Rx, treatment.

${ }^{a}$ Comparison of control (lactulose) and experimental (PEG) groups using Wilcoxon (Mann-Whitney) rank sum for ammonia and HESA, Kaplan-Meier for length of stay, and Fisher's exact test for categorical variables.

b24-hour HESA on 23 of 25 patients: One was competent and refused testing; one was discharged in $<24$ h; initial HESA was omitted from analysis.

were no significant changes in serum sodium, creatinine, or BUN after either PEG or lactulose treatment. Additionally, when the 25 subjects with dehydration and HE were analyzed, PEG remained superior to lactulose for the primary outcome (lactulose HESA: baseline $=2.2 \pm 0.9[M \pm \mathrm{SD}], 24$ hours $=1.5$ $\pm 0.8, n=15$; PEG HESA: baseline $=2.4 \pm 1.0,24$ hours $=0.7$ $\pm 0.9, n=10 ; p=0.013$, Spearman). ${ }^{37}$

The data suggest that bowel cleansing with PEG (normally used for colonoscopy preparations) appears to be a safe and effective alternative to treat acute/overt HE compared with lactulose. Moreover, and perhaps most importantly, it has the potential to decrease hospital length of stay. Because PEG has a transient effect, follow-up therapy with lactulose to prevent recurrent $\mathrm{HE}$ is important.

\section{Conclusions}

Previous work on ammonia in HE set the groundwork for a series of recent trials focused on reduction of ammonia in patients with HE in cirrhotic patients. Evolving evidence suggests that some of these novel approaches will be effective. Polyethylene glycol 3350 is extremely attractive for use in hospitalized patients with acute overt HE. Further research is still required to determine optimal dosing and duration of therapy. Ammonia scavengers including ornithine phenylacetate and GPB appear to be promising, while liver dialysis machines may have future use beyond the research spectrum if costs are reduced and outcomes show long-term benefits.

\section{Abbreviations}

AG anion gap

BDL bile-duct ligation

BUN blood urea nitrogen

CHE covert hepatic encephalopathy

$\mathrm{CI}$ confidence interval

GI gastrointestinal

GPB glycerol phenylbutyrate

HE hepatic encephalopathy
HESA hepatic encephalopathy scoring algorithm

HR hazard ratio

ITT intention to treat

$M \quad$ mean

MELD Model for End Stage Liver Disease

MARS molecular adsorbent recirculating system

OP ornithine phenylacetate

PAA phenylacetate

PAGN phenylacetylglutamine

PEG polyethylene glycol 3350-electrolyte solution

RBANS Repeatable Battery for the Assessment of Neuropsychological Status

RR risk ratio

SMT standard medical therapy

UGIB upper gastrointestinal bleeding

UCD urea cycle disorders.

\section{Acknowledgments}

Robert S. Rahimi receives research support from Ocera Therapeutics. Don C. Rockey receives research support from the NIH, Gilead Sciences, Hyperion, Ocera, and Galectin Therapeutics.

\section{References}

1 Wong RJ, Gish RG, Ahmed A. Hepatic encephalopathy is associated with significantly increased mortality among patients awaiting liver transplantation. Liver Transpl 2014;20(12): 1454-1461

2 Blei AT, Córdoba J; Practice Parameters Committee of the American College of Gastroenterology. Hepatic encephalopathy. Am J Gastroenterol 2001;96(7):1968-1976

3 Vince AJ, Burridge SM. Ammonia production by intestinal bacteria: the effects of lactose, lactulose and glucose. J Med Microbiol 1980; 13(2):177-191

4 Floch MH, Katz J, Conn HO. Qualitative and quantitative relationships of the fecal flora in cirrhotic patients with portal systemic 
encephalopathy and following portacaval anastomosis. Gastroenterology 1970;59(1):70-75

5 Wolpert E, Phillips SF, Summerskill WH. Ammonia production in the human colon. Effects of cleansing, neomycin and acetohydroxamic acid. N Engl J Med 1970;283(4):159-164

6 Plauth M, Roske AE, Romaniuk P, Roth E, Ziebig R, Lochs H. Postfeeding hyperammonaemia in patients with transjugular intrahepatic portosystemic shunt and liver cirrhosis: role of small intestinal ammonia release and route of nutrient administration. Gut 2000;46(6):849-855

7 Olde Damink SW, Jalan R, Redhead DN, Hayes PC, Deutz NE, Soeters PB. Interorgan ammonia and amino acid metabolism in metabolically stable patients with cirrhosis and a TIPSS. Hepatology 2002; 36(5):1163-1171

8 Romero-Gómez M, Ramos-Guerrero R, Grande L, et al. Intestinal glutaminase activity is increased in liver cirrhosis and correlates with minimal hepatic encephalopathy. J Hepatol 2004;41(1):49-54

9 Romero-Gómez M, Jover M, Del Campo JA, et al. Variations in the promoter region of the glutaminase gene and the development of hepatic encephalopathy in patients with cirrhosis: a cohort study. Ann Intern Med 2010;153(5):281-288

10 Häussinger D, Kircheis G, Fischer R, Schliess F, vom Dahl S. Hepatic encephalopathy in chronic liver disease: a clinical manifestation of astrocyte swelling and low-grade cerebral edema? J Hepatol 2000; 32(6):1035-1038

11 Ghabril M, Zupanets IA, Vierling J, et al. Glycerol phenylbutyrate in patients with cirrhosis and episodic hepatic encephalopathy: a pilot study of safety and effect on venous ammonia concentration. Clin Pharm Drug Dev 2013;2:278-284

12 Rockey DC, Vierling JM, Mantry P, et al; HALT-HE Study Group. Randomized, double-blind, controlled study of glycerol phenylbutyrate in hepatic encephalopathy. Hepatology 2014;59(3):1073-1083

13 Lee B, Rhead W, Diaz GA, et al. Phase 2 comparison of a novel ammonia scavenging agent with sodium phenylbutyrate in patients with urea cycle disorders: safety, pharmacokinetics and ammonia control. Mol Genet Metab 2010;100(3):221-228

14 McGuire BM, Zupanets IA, Lowe ME, et al. Pharmacology and safety of glycerol phenylbutyrate in healthy adults and adults with cirrhosis. Hepatology 2010;51(6):2077-2085

15 Diaz GA, Krivitzky LS, Mokhtarani M, et al. Ammonia control and neurocognitive outcome among urea cycle disorder patients treated with glycerol phenylbutyrate. Hepatology 2013;57(6):2171-2179

16 Lichter-Konecki U, Diaz GA, Merritt JL II, et al. Ammonia control in children with urea cycle disorders (UCDs); phase 2 comparison of sodium phenylbutyrate and glycerol phenylbutyrate. Mol Genet Metab 2011;103(4):323-329

17 Smith W, Diaz GA, Lichter-Konecki U, et al. Ammonia control in children ages 2 months through 5 years with urea cycle disorders: comparison of sodium phenylbutyrate and glycerol phenylbutyrate. J Pediatr 2013;162(6):1228-1234, 1234.e1

18 Jalan R, Wright G, Davies NA, Hodges SJ. L-Ornithine phenylacetate (OP): a novel treatment for hyperammonemia and hepatic encephalopathy. Med Hypotheses 2007;69(5):1064-1069

19 Ventura-Cots M, Arranz JA, Simón-Talero M, et al. Safety of ornithine phenylacetate in cirrhotic decompensated patients: an open-label, dose-escalating, single-cohort study. J Clin Gastroenterol 2013;47(10):881-887

20 Córdoba J, Ventura-Cots M. Drug-induced removal of nitrogen derivatives in urine: a new concept whose time has come. Hepatology 2014;59(3):764-766
21 Shoji T, Wada A, Inoue K, et al. Prospective randomized study evaluating the efficacy of the spherical adsorptive carbon AST-120 in chronic kidney disease patients with moderate decrease in renal function. Nephron Clin Pract 2007;105(3):c99-c107

22 Schulman G, Agarwal R, Acharya M, Berl T, Blumenthal S, Kopyt N. A multicenter, randomized, double-blind, placebo-controlled, dose-ranging study of AST-120 (Kremezin) in patients with moderate to severe CKD. Am J Kidney Dis 2006;47(4):565-577

23 Sanaka T, Sugino N, Teraoka S, Ota K. Therapeutic effects of oral sorbent in undialyzed uremia. Am J Kidney Dis 1988;12(2):97-103

24 Owada A, Nakao M, Koike J, Ujiie K, Tomita K, Shiigai T. Effects of oral adsorbent AST-120 on the progression of chronic renal failure: a randomized controlled study. Kidney Int Suppl 1997; 63:S188-S190

25 Shen B, Pardi DS, Bennett AE, et al. The efficacy and tolerability of AST-120 (spherical carbon adsorbent) in active pouchitis. Am J Gastroenterol 2009;104(6):1468-1474

26 Hiraishi M. The effect of oral adsorbent on surgically induced hepatic failure. Jpn J Surg 1987;17(6):517-527

27 Pockros P, Hassanein T, Vierling J, et al. Phase 2, multicenter, randomized study of ast-120 (spherical carbon adsorbent) vs. lactulose in the treatment of low-grade hepatic encephalopathy (HE). J Hepatol 2009;50:S43-S44

28 Bosoi CR, Parent-Robitaille C, Anderson K, Tremblay M, Rose CF. AST-120 (spherical carbon adsorbent) lowers ammonia levels and attenuates brain edema in bile duct-ligated rats. Hepatology 2011; 53(6):1995-2002

29 Kundra A, Jain A, Banga A, Bajaj G, Kar P. Evaluation of plasma ammonia levels in patients with acute liver failure and chronic liver disease and its correlation with the severity of hepatic encephalopathy and clinical features of raised intracranial tension. Clin Biochem 2005;38(8):696-699

30 Palm K, Luthman K, Ros J, Grasjo J, Artursson P. Effect of molecular charge on intestinal epithelial drug transport: $\mathrm{pH}$-dependent transport of cationic drugs. J Pharmacol Exp Ther 1999;291(2): 435-443

31 Bajaj JS, Sheikh MY, Chojkier M, et al. AST-120 (spherical carbon adsorbent) in covert hepatic encephalopathy: results of the astute trial. J Hepatol 2013;58(5):S84

32 Hassanein TI, Tofteng F, Brown RS Jr, et al. Randomized controlled study of extracorporeal albumin dialysis for hepatic encephalopathy in advanced cirrhosis. Hepatology 2007; 46(6):1853-1862

33 Hill K, Hu KQ, Cottrell A, Teichman S, Hillebrand DJ. Charcoal-based hemodiabsorption liver support for episodic type $C$ hepatic encephalopathy. Am J Gastroenterol 2003;98(12):2763-2770

34 Tsipotis E, Shuja A, Jaber BL. Albumin dialysis for liver failure: a systematic review. Adv Chronic Kidney Dis 2015;22(5): 382-390

35 Rahimi RS, Singal AG, Cuthbert JA, Rockey DC. Lactulose vs polyethylene glycol 3350-electrolyte solution for treatment of overt hepatic encephalopathy: the HELP randomized clinical trial. JAMA Intern Med 2014;174(11):1727-1733

36 Hammer HF, Santa Ana CA, Schiller LR, Fordtran JS. Studies of osmotic diarrhea induced in normal subjects by ingestion of polyethylene glycol and lactulose. J Clin Invest 1989;84(4): 1056-1062

37 Rahimi RS, Cuthbert JA, Rockey DC. Lactulose vs polyethylene glycol for treatment of hepatic encephalopathy: reply.JAMA Intern Med 2015;175(5):868-869 